\title{
ESTUDO DA CINÉTICA E DO EQUILÍBRIO DE ADSORÇÃO DOS CORANTES AZUL TURQUESA QG E AMARELO REATIVO 3R EM ÇARVÃO ATIVADO
}

\author{
Carlos Eduardo Borba ${ }^{1}$ \\ Aparecido Nivaldo Módenes \\ Fernando Rodolfo Espinoza-Quiñones \\ Fernando Henrique Borba \\ André Fernandes Bassi \\ Caroline Ribeiro
}

\begin{abstract}
Resumo: Neste trabalho, foi avaliado o desempenho do carvão ativado na adsorção dos corantes azul turquesa QG e amarelo reativo 3R. Para tanto, foram obtidos dados experimentais da cinética e do equilíbrio de adsorção para cada corante individualmente. Os experimentos foram conduzidos em sistema batelada sob temperatura e agitação controlada. Para descrever o equilíbrio de adsorção foi utilizado o modelo de isoterma de Langmuir. Para descrever a cinética de adsorção foram utilizados modelos matemáticos baseados nos mecanismos de transferência de massa que ocorrem no processo de adsorção (difusão no filme externo, adsorção na superfície, difusão nos poros). Os dados experimentais de equilíbrio mostraram que o processo de adsorção dos corantes pelo carvão ativado é favorável, sendo que a isoterma de Langmuir descreveu satisfatoriamente o equilíbrio de adsorção dos sistemas investigados. Os dados experimentais da cinética de adsorção mostraram que o tempo de equilíbrio foi de 800 e 1200 min para os corantes azul e amarelo, respectivamente. Na descrição da cinética de adsorção, os três modelos testados representaram de forma eficiente o processo.
\end{abstract}

Palavras-chaves: Adsorção, Equilíbrio, Cinética, Corante têxtil.

Abstract: In this work, the performance of activated carbon on adsorption of the dyes turquoise blue and reactive yellow was evaluated. Equilibrium and kinetic experimental data for each dye individually were obtained. The experiments were conducted in batch system under controlled temperature and agitation. To describe the equilibrium, the Langmuir isotherm was used. To describe the adsorption kinetics, the mathematical models based on mass transfer mechanisms diffusion in the film external, surface adsorption and pore diffusion were used. The equilibrium experimental data showed that the adsorption of dyes by activated carbon is favorable. The Langmuir isotherm described satisfactorily the adsorption equilibrium of the systems investigated. The kinetics experimental data showed that the equilibrium time is 800 and 1200 min for the blue and yellow dyes, respectively. The adsorption kinetics was described satisfactorily by the three models tested.

Keywords: Adsorption, Equilibrum, Kinetics, Textil dye.

\footnotetext{
1 Programa de Pós-graduação Stricto Sensu em Engenharia Química da Universidade Estadual do Oeste do Paraná - UNIOESTE. Rua da Faculdade, 645. Jardim La Salle. CEP 85902-000. E-mail:borba_deq@yahoo.com.br.
} 


\section{INTRODUÇÃO}

A presença de corantes têxteis em águas residuais industriais provoca graves problemas ao meio ambiente, pois a maioria são compostos orgânicos tóxicos e altamente resistentes à degradação. Os corantes têxteis sintéticos possuem estruturas moleculares aromáticas formadas por hidrocarbonetos tais como benzeno, naftaleno, antraceno, tolueno e xileno (Gong et al., 2006). Estima-se que 10-20\% dos corantes permanecem em águas residuais (Li et al., 2009).

Os métodos convencionais utilizados na remoção de corantes de águas residuais são a adsorção (Li et al., 2009; Greluk e Hubicki, 2010), floculação (Fang et al., 2010), a oxidação (Dukkanci et al., 2010), a eletrólise (Ruan et al., 2010) e métodos biológicos (Kornaros e Lyberatos, 2006). Os processos de tratamento biológicos, tais como lagoas aerada, são eficientes na remoção de sólidos suspensos mas são ineficazes na remoção de cor destas águas residuais (Meyer et al., 2002). A floculação, a oxidação e a eletrólise são eficientes na remoção de cor, no entanto, enquanto a floculação necessita de adição de reagentes, a oxidação e a eletrólise podem formar, pela quebra dos compostos orgânicos formadores dos corantes sintéticos, compostos mais tóxicos que os compostos originais, como por exemplo, organoclorados. Desta forma, a aplicação do processo de adsorção vêm sendo estudada, para a remoção de corantes de águas residuais.

Os sistemas de adsorção podem ser operados em regime batelada ou contínuo. No sistema batelada são, geralmente, empregados tanques de mistura, enquanto que no sistema contínuo são utilizadas colunas de leito fixo que operam por meio de ciclos de regeneração.

Em geral, estudos cinéticos e de equilíbrio são realizados em sistema batelada. Para tanto, é utilizado procedimento padrão, que consiste em misturar um volume fixo de solução com uma quantidade conhecida de adsorvente, em condições controladas (tempo de contato, taxa de agitação, temperatura e $\mathrm{pH}$ ). Em tempos pré-determinados, a concentração residual do corante em solução é medida (Crini e Badot, 2008). Estudos em sistema batelada podem ser úteis na investigação do processo em sistema contínuo (Jena et al., 2004), pois juntamente com a modelagem matemática, permitem a obtenção de parâmetros de transferência de massa e de equilíbrio. Além disto, a utilização de modelos matemáticos auxiliam na identificação dos mecanismos envolvidos no processo, na análise e interpretação de dados experimentais, na predição de respostas a mudanças de condições de operação e na otimização dos processos (Volesky, 2001).

A construção de um modelo matemático que represente a dinâmica de adsorção em sistema batelada requer um balanço de massa no sistema, equaçôes que representem apropriadamente dos dados de equilíbrio e equações que representem os efeitos de transferência de massa que ocorrem no processo.

Os mecanismos de transferência de massa presentes no processo de adsorção são os seguintes: 1) difusão do soluto do seio da fase fluida para a superfície do adsorvente (difusão no filme líquido estagnado); 2) Adsorção do soluto na superfície do adsorvente; 3) Difusão do soluto nos poros do adsorvente. Estas etapas estão ilustradas na Figura 1.

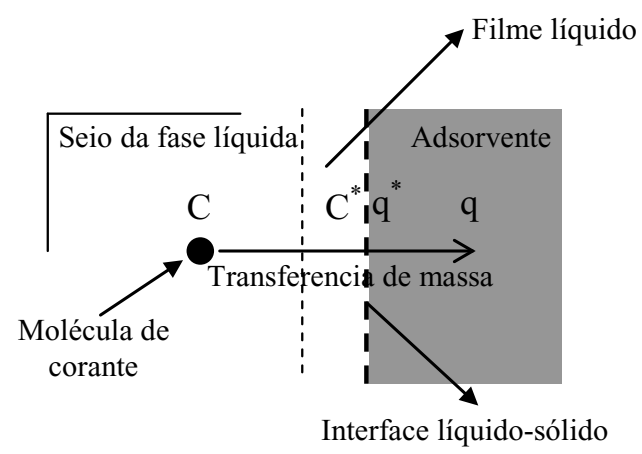

Figura 1. Representação dos mecanismos de transferência de massa envolvidos no processo de adsorção

Desta forma, este trabalho tem como objetivo estudar a cinética e o equilíbrio de adsorção, em sistema monocomponente, dos corantes azul turquesa QG e amarelo reativo, utilizando como adsorvente carvão ativado.

\section{MATERIAIS E MÉTODOS}

\subsection{MATERIAIS}

Os materiais utilizados neste trabalho foram os seguintes:

- Carvão ativado fornecido pela empresa Guaramex;

- Corantes azul turquesa QG e amarelo reativo 3R fornecidos pela empresa Texpal Química S/A; 
- Água destilada;

- Ácido clorídrico $1 \mathrm{M}$.

\subsection{PREPARO DOS REAGENTES}

Os testes de adsorção dos corantes azul turquesa e amarelo reativo pelo carvão ativado foram realizados utilizando efluente sintético preparado a partir da dissolução do corante em pó em água destilada. $\mathrm{O} \mathrm{pH}$ inicial das soluçôes de corantes foram ajustadas em 2 utilizando $\mathrm{HCl} 1 \mathrm{M}$. Os demais reagentes utilizados neste trabalho tiveram grau pureza analítica.

\subsection{PROCEDIMENTO EXPERIMENTAL}

Os ensaios de adsorção foram realizados para os sistemas monocomponentes: corante azul turquesa - carvão ativado e corante amarelo reativo - carvão ativado.

\subsubsection{Dados experimentais da cinética de} adsorção

Os experimentos da cinética de adsorção dos corantes pelo carvão ativado foram realizados em sistema batelada. Frascos erlenmeyers contendo a solução e o adsorvente foram mantidos sob agitação, em "shaker", e temperatura controlada de $30^{\circ} \mathrm{C}$. Na Figura 2 é apresentado o equipamento utilizado nos ensaios. Em intervalos de tempo pré-determinados os frascos erlenmeyers foram retirados. As amostras foram centrifugadas e analisadas em relação à concentração do corante na solução utilizando espectrofotometria UV/VIS.

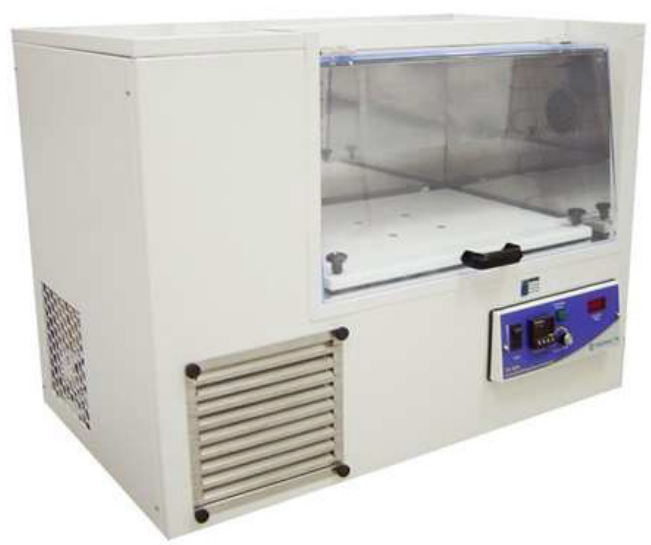

Figura 2. Incubadora refrigerado com agitação (shaker) utilizado nos experimentos
A concentração do corante no adsorvente $(q(t))$, em um instante de tempo $t$, foi calculada pela Equação 1.

$$
q(t)=\frac{V\left(C_{0} \quad C(t)\right)}{m}
$$

Em que $V$ é o volume da solução $\mathrm{L}, C_{0}$ é a concentração inicial da solução $\mathrm{mg} \mathrm{L}^{-1}, C(t)$ é a concentração da soluçãao em um instante de tempo $t \mathrm{mg} \mathrm{L}^{-1} \mathrm{e} m$ é a massa de adsorvente seca g.

\subsubsection{Dados experimentais do equilíbrio de} adsorção

Os dados experimentais de equilíbrio foram realizados em sistema batelada. Frascos erlenmeyer contendo a solução e o adsorvente foram mantidos sob agitação constante em "shaker", com temperatura controlada em $30^{\circ} \mathrm{C}$. O tempo de duração dos ensaios de equilíbrio foi de 1200 min para o corante amarelo e 600 min para o corante azul. Estes valores do tempo de equilíbrio foram baseados nos teste cinéticos. Ao final de cada ensaio, a solução foi separada do adsorvente por meio de centrifugação. As concentrações, inicial e de equilíbrio, do corante na solução em cada frasco foram determinadas por espectrofotometria UV/VIS.

A concentração do corante no adsorvente, no equilíbrio, foi calculada pela Equação 2.

$$
q_{e q}=\frac{V\left(C_{0}-C_{e q}\right)}{m}
$$

Em que $C_{e q}$ é a concentração do corante na fase líquida no equilíbrio em $\mathrm{mg} \mathrm{L}^{-1}, q_{e q}$ é a concentração do corante no adsorvente no equilíbrio em $\mathrm{mg} \mathrm{g}^{-1}$.

\section{MODELAGEM MATEMÁTICA}

A modelagem matemática apresentada neste trabalho compreendeu a modelagem matemática do equilíbrio e da cinética de adsorção.

\subsection{MODELAGEM MATEMÁTICA DO EQUILÍBRIO DE ADSORÇÃO}

Para descrever o equilíbrio de adsorção foi utilizado o modelo de isoterma de adsorção de Langmuir. A isoterma de Langmuir é um modelo 
teórico cuja expressão matemática fundamenta-se nas seguintes hipóteses: (i) todos os sítios do adsorvente têm a mesma atividade para a adsorção; (ii) não existe interação entre as moléculas adsorvidas; (iii) toda adsorção segue o mesmo mecanismo, e cada adsorvente complexo têm a mesma estrutura; (iv) a extensão da adsorção não é mais que a formação de uma camada monomolecular sobre a superfície do adsorvente.

A expressão matemática para isoterma de Langmuir para um sistema monocomponente é dada pela Equação 3 .

$$
q_{e q}=\frac{q_{\max } b C_{e q}}{1+b C_{e q}}
$$

Em que $q_{\max }$ é a concentração máxima do corante adsorvido em $\mathrm{mg} \mathrm{g}^{-1}$ e $b$ é a constante de equilíbrio de adsorção de Langmuir em $\mathrm{L} \mathrm{mg}^{-1}$.

As constantes da isoterma de Langmuir têm significado físico. O parâmetro $b$ representa a razão entre a taxa de adsorção e dessorção. Portanto, elevados valores deste parâmetro indicam forte afinidade do íon pelos sítios do material, enquanto que o parâmetro $q_{\max }$ representa o número total de sítios disponíveis no material adsorvente.

\subsection{MODELAGEM MATEMÁTICA DA CINÉTICA DE ADSORÇÃO}

As equações da taxa cinética são funções matemáticas que descrevem a taxa de variação da concentração em relação ao tempo de uma dada espécie no adsorvente. Fisicamente, estas equações representam a etapa controladora do processo de transferência de massa.

Para representar a taxa de transferência de massa no filme líquido externo foi utilizado a Equação 4.

$$
\frac{d q}{d t}=\frac{K_{F}}{\rho_{S}}\left(C-C_{e q}\right)
$$

Em que $q$ é a concentração do corante no adsorvente $\mathrm{mg} \mathrm{g}^{-1}, C$ é a concentração do corante na fase líquida $\mathrm{mg} \mathrm{L}^{-1}, K_{F}$ é o coeficiente de transferência de massa no filme externo $\min ^{-1}$ e $\rho_{S}$ é a densidade do adsorvente $\mathrm{g} \mathrm{L}^{-1}$.

Neste caso, temos a concentração de equilíbrio na fase líquida $\left(C_{e q}\right)$ calculada, a partir da iso- terma de Langmuir, como função da concentração no adsorvente $(q)$. Assim, a Equação 4 pode ser escrita como:

$$
\frac{d q}{d t}=\frac{K_{F}}{\rho_{S}}\left(C-\left(-\frac{q}{b\left(q-q_{\max }\right)}\right)\right)
$$

Para representar a etapa de adsorção na superfície do adsorvente foi utilizado o modelo cinético de adsorção-dessorção de Langmuir, o qual é representado pela Equação 6.

$$
\frac{d q}{d t}=k_{a d s} C\left(q_{\max }-q\right)-k_{d e s} q
$$

Em que $k_{\text {ads }}$ é a constante cinética de adsorção $\mathrm{g} \mathrm{mg}^{-1} \mathrm{~L}^{-1}, k_{\text {des }}$ é a constante cinética de dessorção.

No equilíbrio $(\boldsymbol{d} / \boldsymbol{d}=0)$, a Equação 6 resulta na isoterma de Langmuir, sendo que o parâmetro $b$ é a razão entre a constante cinética de adsorção e constante cinética de dessorção. Desta forma, a Equação 6 pode ser escrita como:

$$
\frac{d q}{d t}=k_{a d s} C\left(q_{\max }-q\right)-\frac{k_{a d s}}{b} q
$$

Para representar a taxa de transferência de massa no adsorvente foi utilizado a Equação 8. O modelo de força motriz linear, representado pela Equação 8, é aproximação da segunda lei de Fick, e foi proposta originalmente por Glueckauf e Coates (1947). A descrição detalhada desta simplificação é apresentada por Cruz et al. (2006).

$$
\frac{d q}{d t}=K_{S}\left(q_{e q}-q\right)
$$

Em que $K_{S}$ é o coeficiente de transferência de massa no adsorvente $\min ^{-1}$.

Neste caso, temos a concentração de equilíbrio no adsorvente $\left(q_{e q}\right)$ calculada, a partir da isoterma de Langmuir, como função da concentração na fase líquida $(C)$. Assim, a Equação 8 pode ser escrita como:

$$
\frac{d q}{d t}=K_{S}\left(\frac{q_{\max } b C}{b C+1}-q\right)
$$


As condições iniciais são representadas pelas Equações 10 e 11.

$$
\begin{aligned}
& C(0)=C_{0} \\
& q(0)=0
\end{aligned}
$$

Contudo, o modelo matemático que considera como etapa controladora da transferência de massa a resistência no filme líquido externo é formado pelas Equações 1, 5, 10 e 11, àquele que considera a etapa de adsorção na superfície é formado pelas Equaçóes 1, 7, 10 e 11 e, finalmente àquele que considera a difusão no adsorvente é formado pelas Equações 1, 9, 10 e 11.

\subsection{PROCEDIMENTO DE IDENTIFICAÇÃO DOS PARÂMETROS DOS MODELOS}

Nos modelos matemáticos propostos para descrever o equilíbrio e a cinética de adsorção, pode-se distinguir dois grupos de parâmetros. No primeiro grupo estão àqueles obtidos experimentalmente $\left(m, V, \rho_{S}\right)$. O segundo inclui àqueles que foram a partir de um ajuste do modelo aos dados experimentais. Os parâmetros da isoterma de Langmuir $\left(q_{\max }, b\right)$ foram obtidos a partir dos dados experimentais de equilíbrio, enquanto os parâmetros dos modelos cinéticos $\left(K_{F}, k_{a d s}, K_{S}\right)$ foram obtidos a partir dos dados experimentais da cinética de adsorção.

Os parâmetros dos modelos foram obtidos durante a busca de um mínimo para seguinte função objetivo:

$$
F_{O B J}=\sum_{j=1}^{n}\left(q_{j}^{\text {exp }}-q_{j}^{\bmod }\right)^{2}
$$

Em que $n$ é o número de dados experimentais, $q_{j}^{\exp }$ e $q_{j}^{\bmod }$ são as concentrações do corante no adsorvente obtida experimentalmente e calculada pelo modelo, respectivamente.

O método de otimização, desenvolvido por Nelder e Mead (1965) foi utilizado. Para resolver as equaçôes diferenciais dos modelos cinéticos foi utilizado o método de Rosenbrock. Os programas foram desenvolvidos utilizando o software Maple®.

\section{RESULTADOS E DISCUSSŌES}

\subsection{EQUILÍBRIO DE ADSORÇÃO}

Os dados experimentais de equilíbrio dos sistemas corante azul turquesa QG - carvão ativado e corante amarelo reativo $3 \mathrm{R}$ - carvão ativado foram obtidos em sistema batelada. Nas Figuras 3 e 4 são apresentadas, respectivamente, as isotermas de adsorção do corante azul e amarelo. Os resultados mostram que a adsorção dos corantes pelo carvão ativado é favorável. Para descrever o equilíbrio foi utilizada a isoterma de Langmuir. $\mathrm{Na}$ Tabela 1 são apresentados os valores dos parâmetros da isoterma obtidos a partir de um ajuste do modelo aos dados experimentais. Os resultados da simulação, apresentados nas Figuras 3 e 4, mostram que o modelo de isoterma de Langmuir descreveu de forma satisfatória o equilíbrio de adsorção dos corantes. Isto pode ser comprovado pelos valores do coeficiente de correlação apresentados na Tabela 1.

De acordo com os valores de $q_{\max }$ apresentados na Tabela 1 , as capacidades máximas de adsorção, na faixa de concentração investigada, foram de 15,06 e $13,98 \mathrm{mg} / \mathrm{g}$ para os corantes azul e amarelo, respectivamente. Schimmel (2008) estudou a adsorção do corante azul turquesa QG utilizando um carvão comercial fornecido pela alphacarbo Ltda. Nas condiçôes de $\mathrm{pH}$ inicial igual a 2 e temperatura de $30^{\circ} \mathrm{C}$ foi obtido um capacidade máxima de $144,75 \mathrm{mg} / \mathrm{g}$.

Tabela 1. Valores dos parâmetros da isoterma

\begin{tabular}{|c|c|}
\hline \multicolumn{2}{|c|}{ Corante azul turquesa QG } \\
\hline \multicolumn{2}{|c|}{ Isoterma de Langmuir } \\
\hline $\begin{array}{c}q_{\max }=15,06 \pm 0,68 \\
b=0,05 \pm 0,01\end{array}$ & $\begin{array}{l}\mathrm{r}^{2}=0,9805 \\
\mathrm{~F}_{\text {obj }}=1,66\end{array}$ \\
\hline \multicolumn{2}{|c|}{ Corante amarelo reativo $3 \mathrm{R}$} \\
\hline $\begin{array}{c}q_{\max }=13,98 \pm 0,37 \\
b=0,11 \pm 0,02\end{array}$ & $\begin{array}{c}\mathrm{r}^{2}=0,9773 \\
\mathrm{~F}_{\text {obj }}=1,34\end{array}$ \\
\hline
\end{tabular}
de Langmuir ajustados aos dados experimentais obtidos em $\mathrm{pH}_{\text {inicial }}=2$ e $\mathrm{T}=30^{\circ} \mathrm{C}$. 


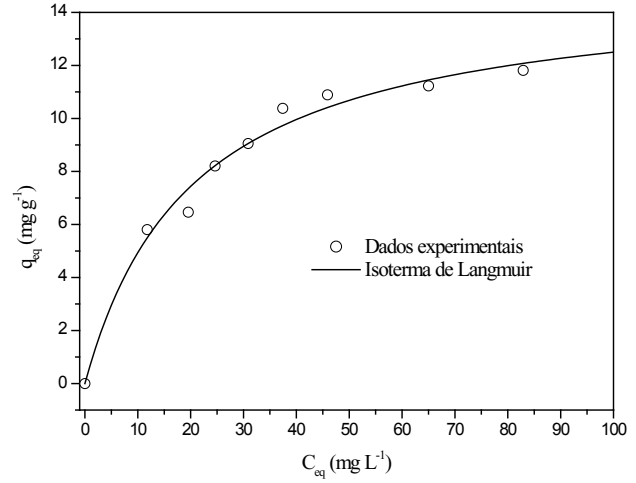

Figura 3. Isoterma de adsorção do corante azul turquesa $\mathrm{QG}$ em $\mathrm{pH}_{\text {inicial }}=2 \mathrm{~T}=30^{\circ} \mathrm{C}$.

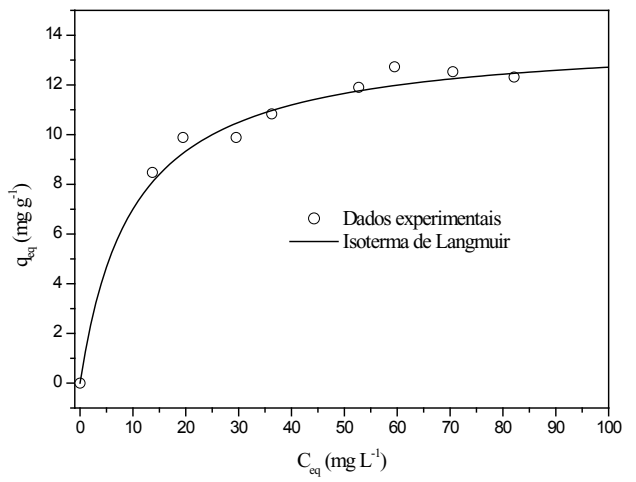

Figura 4. Isoterma de adsorção do corante amarelo reativo $3 \mathrm{R} \mathrm{em} \mathrm{pH}_{\text {inicial }}=2 \mathrm{~T}=30^{\circ} \mathrm{C}$.

\subsection{CINÉTICA DE ADSORÇÃO}

Os dados experimentais da cinética de adsorção dos corantes azul e amarelo são apresentados, respectivamente nas Figuras 5 e 6 . Os resultados mostram que o tempo de equilíbrio foi de aproximadamente $800 \mathrm{~min}$, para o corante azul, e 1200 min para o corante amarelo. Baseado nos valores inicial e final da concentração dos corantes na solução foi obtido uma taxa de remoção de 67 e $82 \%$ para os corantes azul e amarelo, respectivamente. Ainda, os resultados mostram uma cinética mais favorável à adsorção do corante azul.

Para descrever a cinética de adsorção foram utilizados três modelos (difusão no filme, adsorção na superfície, difusão no adsorvente). Os valores dos parâmetros dos modelos ajustados aos dados experimentais cinéticos são apresentados na Tabela 2. Baseado, nos resultados da simulação (ver Figs. 5 e 6) e nos valores da função objetivo (ver Tabela 2) percebe-se que todos os modelos descrevem de forma análoga a cinética de adsorção dos corantes investigados. Assim, nas condi- ções em que os experimentos foram realizados, não foi possível identificar o mecanismo de transferência de massa que controla o processo, sendo neste caso, possível utilizar qualquer um dos 3 modelos para descrever a cinética de adsorção dos corantes azul turquesa QG e amarelo reativo 3F pelo carvão ativado.

Tabela 2. Valores dos parâmetros dos modelos cinéticos ajustados aos dados experimentais obtidos em $\mathrm{pH}_{\text {inicial }}=2$ e $\mathrm{T}=30^{\circ} \mathrm{C}$.

\begin{tabular}{|c|c|}
\hline \multicolumn{2}{|c|}{ Corante azul turquesa QG } \\
\hline \multicolumn{2}{|c|}{ Difusão no filme líquido } \\
\hline$K_{F}=0,0417 \pm$ & $\begin{array}{c}\mathrm{r}^{2}=0,9048 \\
\mathrm{~F}_{\mathrm{obj}}=4,92\end{array}$ \\
\hline \multicolumn{2}{|c|}{ Adsorção na superfície } \\
\hline$k_{a d s}=0,0031 \pm$ & $\begin{array}{c}\mathrm{r}^{2}=0,8982 \\
\mathrm{~F}_{\text {obj }}=4,32\end{array}$ \\
\hline \multicolumn{2}{|c|}{ Difusão no adsorvente } \\
\hline$K_{S}=0,0384 \pm$ & $\begin{array}{c}\mathrm{r}^{2}=0,9098 \\
\mathrm{~F}_{\text {obj }}=5,47\end{array}$ \\
\hline \multicolumn{2}{|c|}{ Corante amarelo reativo 3R } \\
\hline \multicolumn{2}{|c|}{ Difusão no filme líquido } \\
\hline$K_{F}=0,0022 \pm$ & $\begin{array}{c}\mathrm{r}^{2}=0,8819 \\
\mathrm{~F}_{\text {obj }}=3,52\end{array}$ \\
\hline \multicolumn{2}{|c|}{ Adsorção na superfície } \\
\hline$k_{a d s}=0,0002 \pm$ & $\begin{array}{c}\mathrm{r}^{2}=0,8755 \\
\mathrm{~F}_{\text {obj }}=3,18\end{array}$ \\
\hline \multicolumn{2}{|c|}{ Difusão no adsorvente } \\
\hline$K_{S}=0,0028 \pm$ & $\begin{array}{c}\mathrm{r}^{2}=0,8860 \\
\mathrm{~F}_{\text {obj }}=3,60\end{array}$ \\
\hline
\end{tabular}

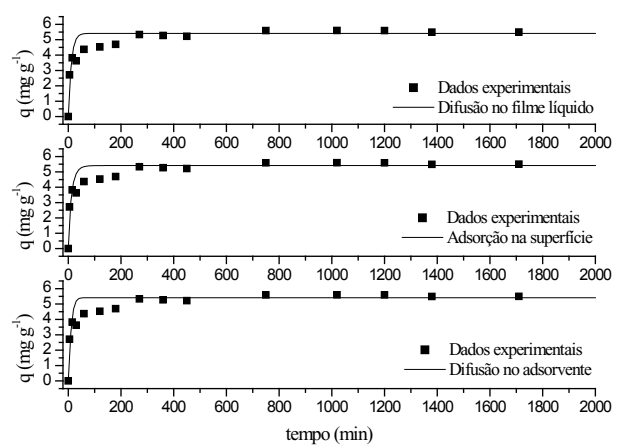

Figura 5. Cinética de adsorção do corante azul turquesa $\mathrm{QG}$ em $\mathrm{pH}_{\text {inicial }}=2 \mathrm{e} \mathrm{T}=30^{\circ} \mathrm{C}$. 


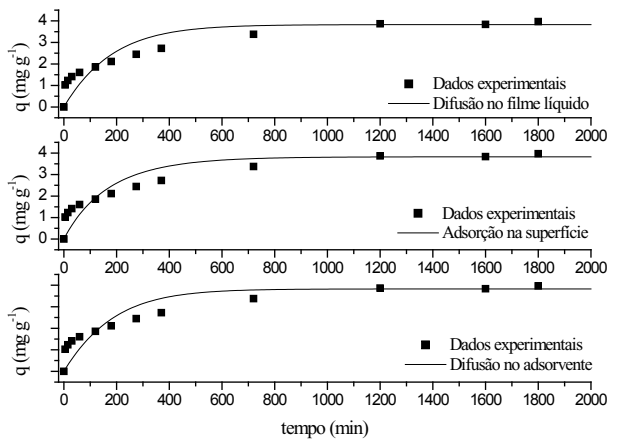

Figura 6. Cinética de adsorção do corante

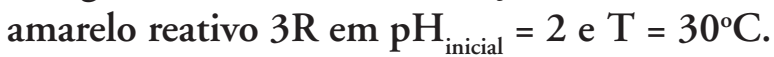

\section{CONCLUSÃO}

Neste trabalho, foram obtidos dados experimentais da cinética e do equilíbrio de adsorção dos sistemas corante azul turquesa QG - carvão ativado e corante amarelo reativo $3 \mathrm{R}$ - carvão ativado. Os dados de equilíbrio mostraram que o processo de adsorção dos corantes pelo carvão ativado utilizado é favorável. Ainda foi verificado que a isoterma de Langmuir descreve satisfatoriamente o equilíbrio do sistema. Os dados cinéticos mostraram que o tempo de equilíbrio foi de 800 e 1200 min para os corantes azul e amarelo, respectivamente. Em adição, foram obtidas taxas de remoção de 67 e $82 \%$ para os corantes azul e amarelo, respectivamente. Finalmente, na descrição da cinética de adsorção, os três modelos testados representaram de forma satisfatória o processo.

\section{REFERÊNCIAS}

Crini, G., Badot. P. M. 2008. Application of chitosan, a natural aminopolysaccharide, for dye removal from aqueous solutions by adsorption processes using batch studies: A review of recent literature. Progress in Polymer Science. 33, pp. 399-447.

Cruz P., Magalhães, F.D., Mendes, A. 2006. Generalized linear driving force approximation for adsorption of multicomponent mixtures. Chemical Engineering Science 61, pp. 3519 - 3531.

Dukkanci, M., Gunduz, G., Yilmaz, S., Prihod'ko, R.V. 2010. Heterogeneous Fentonlike degradation of rhodamine $6 \mathrm{G}$ in water using CuFeZSM-5 zeolite catalyst prepared by hydrothermal synthesis. Journal of Hazardous Materials. 181, pp. 343-350.
Fang, R., Cheng, X., Xu, X. 2010. Synthesis of lignin-base cationic flocculant and its application in removing anionic azo-dyes from simulated wastewater. Bioresource Technology. 101, pp. 7323-7329.

Glueckauf, E., Coates, J. J. 1947. Theory of Chromatography Part IV: The influence of incomplete equilibrium on the front boundary of chromatograms and on the effectiveness of separation, Journal of American Chemical Society, pp. 1315-1321.

Gong, R., Zhang, X., Liu, H., Sun, Y., Liu, B. 2007. Uptake of cationic dyes from aqueous solution by biosorption onto granular kohlrabi peel. Bioresource Technology. 98, pp. 1319 - 1323.

Greluk, M., Hubicki, Z. 2010. Kinetics, isotherm and thermodynamic studies of Reactive Black 5. Chemical Engineering Journal. 162, pp. 919-926

Jena, P. R., Basu, J. K., De, S. 2004. A generalized shrinking core model for multicomponent batch adsorption processes. Chemical Engineering Journal. 102, pp. 267-275.

Kornaros, M., Lyberatos, G. 2006. Biological treatment of wastewaters from a dye manufacturing company using a trickling filter. Journal of Hazardous Materials. 136, pp. 95-102.

Li, Q., Yue, Q.Y., Su, Y., Gao, B.Y., Li, J. 2009. Two-step kinetic study on the adsorption and desorption of reactive dyes at cationic polymer/ bentonite. Journal of Hazardous Materials. 165, pp. $1170-1178$.

Meyer, V., Carlsson, F.H.H., Oellermann, R.A. 1992. Decolourization of textile effluent using a low cost natural adsorbent material. Water Science Technology. 26(5-6), pp.1205-1211.

Nelder J. A., Mead R. 1965. A simplex method for function minimization. The Computer Journal. 7, pp. 308-315.

Ruan, X.C., Liu, M.Y., Zeng, Q.F., Ding, Y.H. 2010. Degradation and decolorization of reactive red $\mathrm{X}-3 \mathrm{~B}$ aqueous solution by ozone integrated with internal microelectrolysis, Separation Purification Technology. 74, pp. 195-201. 
Schimmel, D. 2008. Adsorção dos corantes azul reativo $5 \mathrm{G}$ e azul turquesa QG em carvão atiçado comercial. Dissertação de Mestrado apresentada na Faculdade de Engenharia Química da Universidade Estadual do Oeste do Paraná.
Volesky, B. 2001. Detoxification of Metal-Bearing Effluents: Biosorption for the Next Century. Hydrometallurgy. 59, pp. 203-216. 